\title{
MOTION JUDGMENT ALGORITHM BASED ON JOINT ANGULAR VELOCITY WITH VARIABLE VISCOELASTIC ASSISTIVE SUIT
}

\author{
RYUJI SUZUKI, SEIGO KIMURA, MANABU OKUI, TARO NAKAMURA \\ Faculty of Science and Engineering, Precision Mechanics, Chuo University, Kasuga 1-13-27, \\ Tokyo, 112-8551, Japan \\ E-mail: ryu_suzuki@bio.mech.chuo-u.ac.jp,s_kimura@bio.mech.chuo-u.ac.jp, \\ manabu.okui48@gmail.com,nakamura@mech.chuo-u.ac.jp \\ RIE NISHIHAMA \\ Research and Development Initiative, Chuo University, Kasuga, \\ Tokyo, 112-8551, Japan \\ E-mail:r_nishihama@bio.mech.chuo-u.ac.jp
}

\begin{abstract}
In recent years, the burden for labor is increasing due to an aging society. To solve this issue, assistive suits are proposed as one of the solutions. In the previous study, an assistive suit has been developed that is based on variable viscoelasticity. However, the motion judgment interface for the variable viscoelastic based actuating device has not been studied. In this study, joint angle and joint angular velocity are used to judge the motion of the wearer, because variable viscoelastic based actuating device has a high backdrivability. In addition, with a suit of high backdrivability, the intention of a wearer can be shown as the joint angle. This study reports the motion judgment algorithm that is based on the wearer's joint angle, and joint angular velocity, and experiments have been conducted to evaluate the utility of the algorithm.
\end{abstract}

\section{Introduction}

Currently, in Japan, the burden for labor is increasing due to an aging society [1]. To solve this issue, assistive suits have been proposed and studied actively [2] - [4]. In the previous study, motors and reduction gears were used as actuators in assistive suits. The suit can assist the wearer with high positioning accuracy. However, in general, as motors have low output density, they tend to be heavy when high outputs are required. Thus, reduction gears are proposed. Reduction gears enable the motors to output an adequate power. However, with reduction gears and motors, the inertia of the output side could be high, and lower the suit's backdrivability. Therefore, assistive suits with motors and reduction gears inhibit the motion of the wearer when assistive suits are not activated. In addition, many sensors and complex controllers are required.

Moreover, pneumatic artificial muscles are also being used as actuators in assistive suits [4]. Artificial muscles are lightweight, flexible, and have high output density. Thus, assistive suits with artificial muscle can output high assistive power with high backdrivability while being lightweight. The suits assist the wearer to move but they do not assist the consecutive motion of the wearer. Additionally, some assistive suits focus on variable elasticity of the artificial muscle, however, these suits do not discuss the assistive effect to the wearer [5].

Springs and dampers are also used in assistive suits [6][7]. Generally, as these suits do not consume a large amount of energy, they can be mobile. However, these suits only output the assistive power in one direction, and there are limitations to the motion.

Due to these reasons, an assistive suit based on variable viscoelasticity has been developed [8]. The characteristic of variable viscoelasticity is inspired by the human joint motion method. In the previous study, variable viscoelasticity is implemented with two elements; straightfiber-type artificial muscle (SFT-AM) and magneto rheological fluid brake (MR brake). SFT- 
AM has variable elasticity and MR brake has variable viscosity. Figure 1 . shows the mechanism of variable viscoelasticity. The following statement has been studied.

1. Assistive effect based on variable viscoelasticity [8]

2. Development of the variable viscoelastic assistive suit "Airsist I" [8]

3. Assistive effect confirmed by measuring the subject's electromyogram (EMG) [9]

4. Proposal of the variable viscoelastic control strategy [10]

However, to expand the practical use of the assistive suit, judgment of wearer's motion should be measured, and an adequate assistive power be output in each motion.

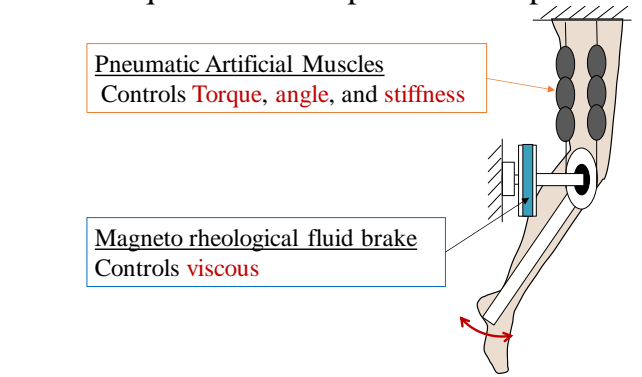

Figure 1. Assistive system based on the variable viscoelasticity

There are several ways to judge the wearer's motion. With EMG of the wearer [11], accelerometers [12] [13], and zero moment point (ZMP) [14]. EMG can pick up the wearer's intention ahead. However, EMG has high sensor noise, and it takes a lot of time to put the electromyograph sensors on wearers. With accelerometers, sensor noise is less and fewer sensors are required [12][13]. In this method, there are various algorithms; however, they are not real time based. ZMP is the method that uses the force reaction of the wearer's sole. There are several methods with ZMP, for instance, an algorithm for the biped walking robot [15]. Sensors should be installed to the shoes, however, it makes the system complex. To make the assistive suit simple, number of sensors and labor when wearing the suit should be reduced and using a real time based system using the joint angle and joint angular velocity is proposed [16]. For example, the method that judges the gait cycle [17].

In this study, the motion judgment algorithm based on the wearer's joint angle and joint angular velocity is proposed. In this method, the joint angle and joint angular velocity is used because the variable viscoelastic assistive device has a high backdrivability. The five types of motions, namely gait, standing up/sitting down, stance with leg opening, sitting, and stance, with the assistive suit are judged.

The study is organized as follows. Section 2 provides the outline of the assistive suit based on variable viscoelasticity. Section 3 provides the motion judgment algorithm with joint angle and joint angular velocity. Section 4 describes the evaluation experiment with the data of joint angle and joint angular velocity that is measured with motion capturing system. Finally, in section 5, conclusion and scope for future works are described.

\section{Assistive suit based on variable viscoelasticity}

Figure. 2 shows the assistive suit "Airsist I" based on variable viscoelasticity. The suit has two elements to implement the variable viscoelasticity which has already been described above. First is the SFT-AM (Figure. 3). An SFT-AM is a pneumatically driven actuator with variable stiffness. The other one is the MR brake (Figure. 4). Inside an MR brake, there is MR fluid, and the viscosity is varied by applying the magnetic field to the MR fluid. With these two elements, variable viscoelasticity is implemented. In addition, antagonized SFT-AMs are connected to the pulleys having different diameters that enables to output the assistive torque. The suit has high backdrivability so the wearer can run with the suit when the suit is not actuated. In addition, there are foot plates that suppress the weight of the suit. 


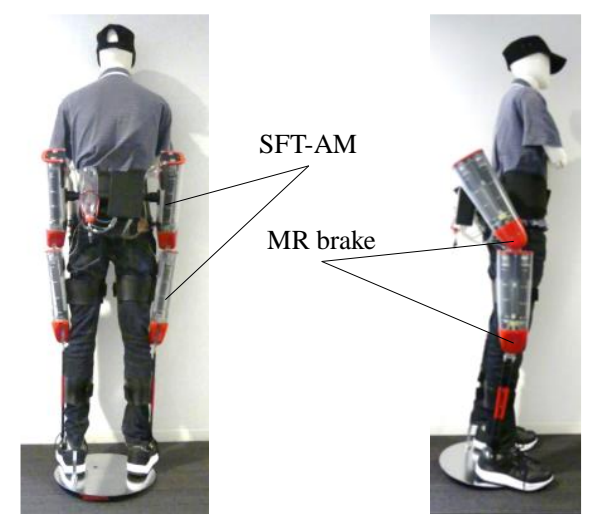

Figure. 2 Concept of variable viscoelastic system

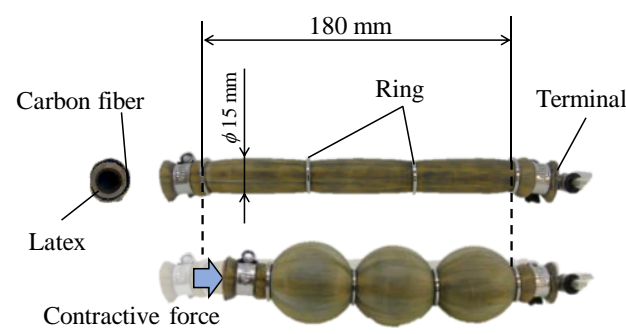

Figure. 3 Straight-fiber-type artificial muscle (SFT-AM)

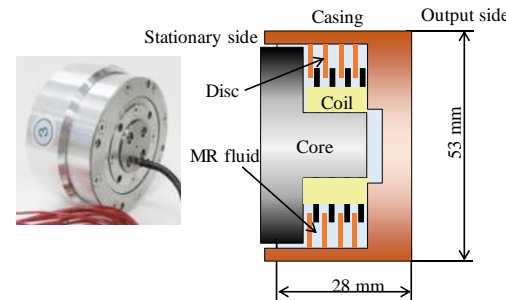

Figure. 4 Magneto rheological fluid brake (MR Brake)

\section{Motion Judgment Algorithm}

In this section, the motion judgment algorithm that uses the joint angle and joint angular velocity is proposed. This algorithm focuses on the high backdrivablity of the variable viscoelastic assistive suit. Figure. 5 shows the flowchart of the motion algorithm. This algorithm judges the five types of motions, namely, gait, standing up/sitting down, stance with leg opening, sitting, and stance. The motion involving movement of the body weight such as gait, standing up/down is defined as dynamically balanced motion (DBM) and the motion without movement of the body weight such as stance is called statically balanced motion (SBM).

This algorithm starts by comparing SBM and DBM with the threshold values of the joint angular velocity. The joint angle and joint angular velocity are measured with encoders that are installed in the suit. Upon comparing the values of joint angle and joint angular velocity with the threshold value, SBM and DBM are judged. First, the method to judge the DBM is described where gait motion and standing up/down motion are judged by checking whether the legs are moving alternatively. Next, the method to judge the SBM is described. Stance with legs opening motion is judged by checking whether both the legs are aligned. Finally, 
sitting and stance are judged by comparing the joint angle with threshold values. In this study, output signal from the algorithm is called judgment signal.

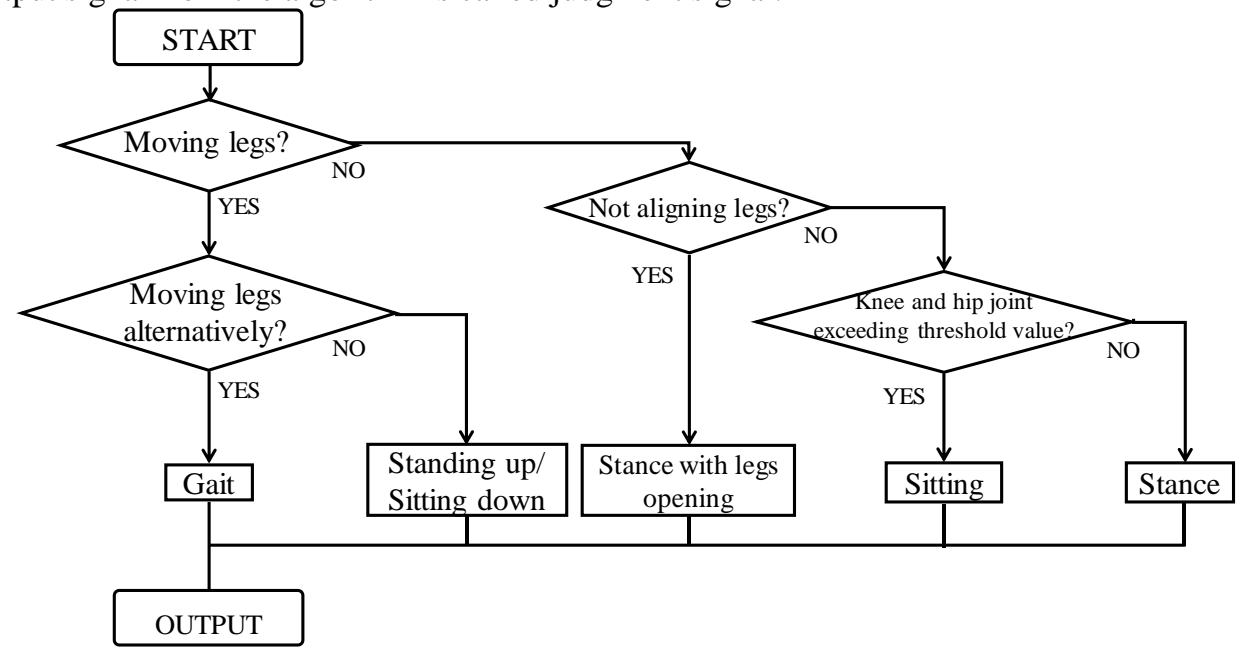

Figure. 5 Motion judgment algorithm

\section{Measurement and evaluation experiment}

The purpose of the evaluation experiment is to verify whether the motion algorithm can judge the gait, stance, stance with legs opening, sitting, and standing up/down. The data of the joint angle and the joint angular velocity are obtained from motion capturing system (MAC 3D system, Motion Analysis Corporation). Adequate instructions for the experiments were given to the subjects on their approval. The minimum joint angle of the subjects was used as the threshold value described above.

\subsection{Measuring method and measurement environment}

To start with, the three motions are measured which are as follows. First is the gait motion (Motion 1). Second is keeping sitting position for $2 \mathrm{~s}$, standing up for $1 \mathrm{~s}$, keeping stance position for $2 \mathrm{~s}$, and walk until the measuring area ends (Motion 2). Third is keeping stance position for $2 \mathrm{~s}$, sitting down for $1 \mathrm{~s}$ and keeping sitting position for $2 \mathrm{~s}$ (Motion 3). Four subjects (Table 1 shows the subjects' fundamental data) enact these three motions. The timing is practiced before the measurement. Additionally, 26 markers for motion capturing were stuck to the subjects. About seven measurement is conducted in each motion. Data with lesser noise are used for the evaluation experiment. In addition, these three motions were selected to confirm the effect of the algorithm when a motion changes to the next motion.

Table 1. Subjects' fundamental data

\begin{tabular}{|c|r|r|r|}
\hline Subject & Height [cm] & Weight $[\mathrm{kg}]$ & Age \\
\hline A & 175 & 64 & 23 \\
\hline B & 167 & 63 & 23 \\
\hline C & 168 & 63 & 23 \\
\hline D & 170 & 55 & 22 \\
\hline
\end{tabular}

Figure. 6 shows the measurement environment. There are eight motion capture cameras (frame rate of $100 \mathrm{fps}$ ) for the upper part of the measurement area. There are three force plates (TF-4060-D, Tec Gihan Co. Ltd., sampling rate of $1000 \mathrm{~Hz}$ ) on the floor. Force plates can measure the reaction force and the moment of the subject's sole. 
In Motion 1, the motion is measured with motion capture cameras as shown in the Figure. 6 (a). Measured data is transferred to the PC through the A/D convertor. In Motion 2 and Motion 3, the chair that makes the subject's knee set at $90^{\circ}$, and the force plate set on the chair, are shown in Figure. 6 (b). After measuring the subjects' joint angle and joint angular velocity, musculoskeletal simulation is run (ver. 1.58, nac Image Technology Inc.), and simulated data is used for the evaluation experiment.

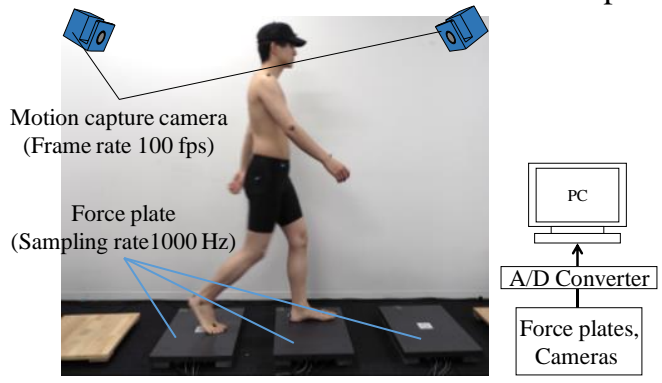

(a) Experimental set up for gait motion

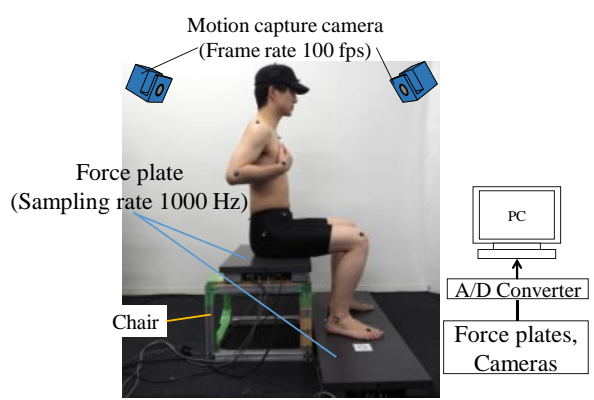

(b) Experimental set up for sitting down, standing up, and gait motion

Figure 6. Experimental set up

\subsection{Evaluation method}

In this evaluation experiment, judgment ratio is used in the evaluation method. Judgment ratio is defined in equation (1).

$$
\frac{t_{\text {judge }}}{t_{\text {motion }}}[\%]
$$

Where, $t_{\text {judge }}[\mathrm{s}]$ is the time taken by the algorithm to output the judgment signal in respond to the motion, and $t_{\text {motion }}[\mathrm{s}]$ is the time of motion we want to measure (for example, when subject stands up for $1 \mathrm{~s}$ while the judgment signal is "stands up" for $0.5 \mathrm{~s}$, and stance position for 0.5 $\mathrm{s}$, the judgment ratio is $50 \%$ for "stands up" motion).

\subsection{Results and discussion}

Table. 2 shows the results of judgment ratio of all subjects in each motion, and Figure. 7 shows the judgment signals of the algorithm. As the characteristic of the results tends to be the same, subject A's results are shown in Figure. 7, and the discussion is based on these.

As shown in Table. 2, the average of the judgment ratio was $81.4 \%$ for Motion 1. In Figure. 7 (a), the judgment signal was "Stance with legs opening" in constant cycle. This is because the joint angular velocity is lower than the threshold value in a constant cycle. When walking, the legs push the floor to keep walking which causes the low joint angular velocity.

In Motion 2, the judgment ratio of the SBM was higher than that of DBM in motions such as standing up or gait motion. As shown in Figure. 7 (b), sitting and stance position satisfy the judgment condition and the judgment signal is appropriate. However, when standing up, the judgment signal was sitting down. The reason for this phenomenon is as follows. When standing up, the subject flexes the joint angle of the hip joint to move the center of gravity. After flexing the hip joint, subject will be in the stance position by extending the knee joint. At this moment, the joint angular velocity of the hip joint is smaller than when moving the center of gravity which causes it to be lower than the threshold values. In addition, deviation of the marker is the reason why the judgment ratio did not achieve a 100\%, as shown in Table. 2 . When shifting to the gait motion, the judgment signal tends to be the same as described in Motion 1, however, is judged as a different motion. 
In Motion 3, the result was same as Motion 2, which means the judgment ratio of SBM was higher than that of DBM.

\begin{tabular}{|c|c|c|c|c|c|c|}
\hline \multicolumn{7}{|l|}{ Motion 1} \\
\hline \multicolumn{2}{|l|}{ Subject } & \multirow{2}{*}{\begin{tabular}{|l|}
$\mathrm{A}$ \\
83.3
\end{tabular}} & \multirow{2}{*}{\begin{tabular}{|l|}
$\mathrm{B}$ \\
83.0
\end{tabular}} & \multirow{2}{*}{\begin{tabular}{|l|} 
C \\
81.8 \\
\end{tabular}} & \multirow{2}{*}{\begin{tabular}{|l|}
$\mathrm{D}$ \\
77.3 \\
\end{tabular}} & \multirow{2}{*}{\begin{tabular}{|l} 
Average \\
81.4
\end{tabular}} \\
\hline Judgment ratio [\%] & Gait & & & & & \\
\hline \multicolumn{7}{|l|}{ Motion 2} \\
\hline \multicolumn{2}{|l|}{ Subject } & $\mathrm{A}$ & $\mathrm{B}$ & $\mathrm{C}$ & $\mathrm{D}$ & Average \\
\hline \multirow[t]{4}{*}{ Judgment ratio [\%] } & Sitting & 95.7 & 100 & 100 & 100 & 98.9 \\
\hline & Standing up & 96.1 & 97.0 & 96.5 & 98.6 & 97.1 \\
\hline & Stance & 100 & 100 & 99.5 & 100 & 99.9 \\
\hline & Gait & 77.2 & 89.7 & 81.8 & 86.1 & 83.7 \\
\hline \multicolumn{7}{|l|}{ Motion 2} \\
\hline \multicolumn{2}{|l|}{ Subject } & A & B & $\mathrm{C}$ & $\mathrm{D}$ & Average \\
\hline \multirow[t]{3}{*}{ Judgment ratio [\%] } & Stance & 100 & 100 & 98.7 & 100 & 100 \\
\hline & Sitting down & 92.6 & 95.8 & 94.5 & 96.4 & 94.8 \\
\hline & Sitting & 98.5 & 98.7 & 100 & 99.4 & 99.2 \\
\hline
\end{tabular}

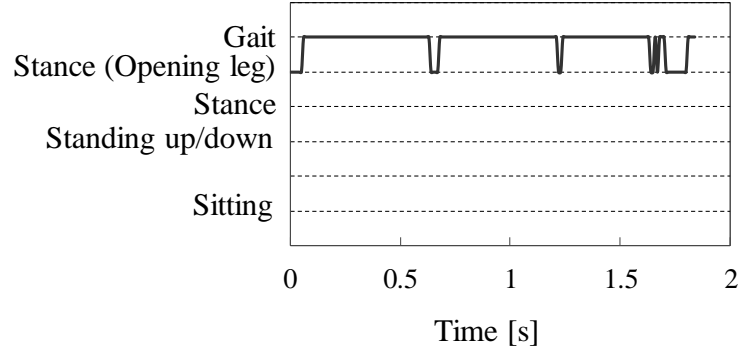

(a) Results of motion judgement of Motion 1

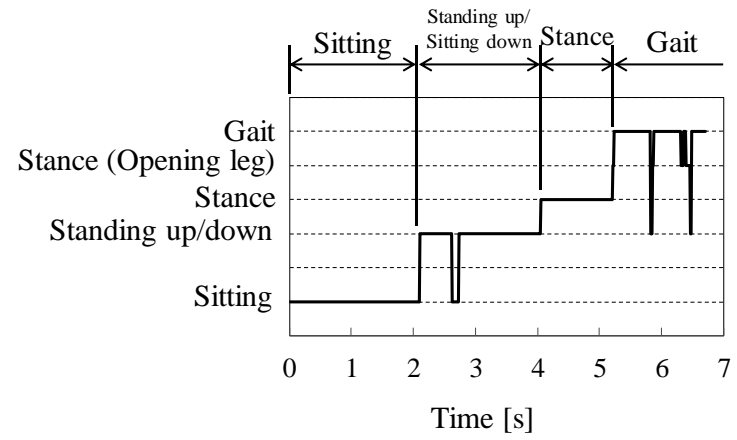

(b) Results of motion judgement of Motion 2

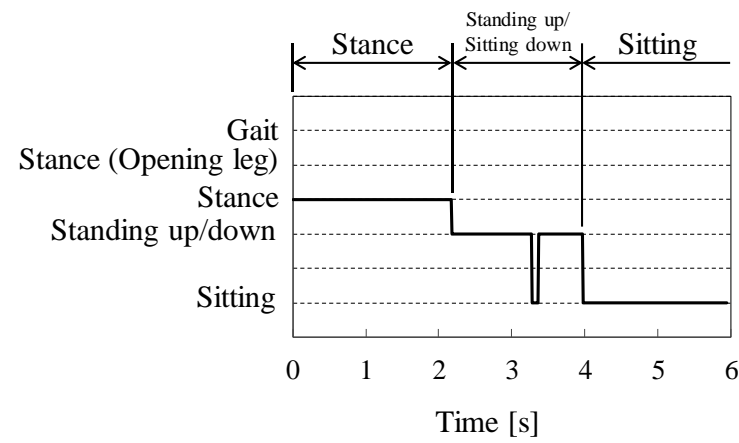

(c) Results of motion judgement of Motion 3

Figure. 7 Results of simulation 


\section{Conclusion and future works}

In this paper, the motion judgment algorithm was proposed based on the joint angle and joint angular velocity. Subjects conducted three motions, and in each motion, the joint angle and joint angular velocity were measured with motion capturing system. With these measured data, the evaluation experiment was conducted based on the proposed algorithm. The judgment ratio of SBM was higher than that of DBM. For future works, the algorithm will be applied to the variable viscoelastic assistive suit (such as Airsist I) and will evaluate the effects of the suit and the wearer.

\section{Acknowledgments}

This research was conducted with the support of the National Institute of Advanced Industrial Science and Technology (NEDO). We would like to thank Editage (www.editage.jp) for English language editing.

\section{References}

1. Ministry of Internal Affairs and Communication, "STATISTICAL HANDBOOK OF JAPAN 2018", pp.13-15, 2018.

2. S. Lee, Y. Sankai: "Power Assist Control for Walking Aid with HAL-3 Based on EMG and Impedance Adjustment around Knee Joint", Intelligent Robots and system, pp.14991504.

3. Curare (AssistMotion Inc.), Available from < http://assistmotion.jp/ >, (Accessed in 4th of March, 2019)

4. Y. Muramatsu, H. Umehara, and H. Kobayashi, "Improvement and Quantitative Performance Estimation of the Back Support Muscle Suit", in Proceedings of $35^{\text {th }}$ Annual International Conference on the IEEE EMBS, Osaka, Japan, July 3-7, 2013, pp. 28442849.

5. D. Maeda, K. Tominaga, T. Oku, Hang T. T. Pham, S. Saeki, M. Uemura, H. Hirai, and F. Miyazaki: "Muscle Synergy Analysis of Human Adaptation to a Variable-Stiffness Exoskeleton: Human Walk with a Knee Exoskeleton with Pneumatic Artificial Muscles", 12th IEEE-RAS International Conference on Humanoid Robots, pp.638-644.

6. Shamaei, K., Napolitano, P.C. and Dollar, A.M.: "Design and functional evaluation of a quasi-passive compliant stance control knee-ankle-foot orthosis", Transactions on Neural Systems and Rehabilitation Engineering, Vol.22, No.2, pp.258-268.

7. Rakunie (MORITA HOLDINGS CORPORATION), Available from https://www.morita1 19.com/en/products/supportwear/rakunie/001.html, (Accessed in $4^{\text {th }}$ of March, 2019)

8. M. Okui, S. Iikawa, Y. Yamada, and T. Nakamura, 2017. "Fundamental characteristic of novel actuation system with variable viscoelastic joints and magneto-rheological clutches for human assistance," Journal of Intelligent Material Sys-tems and Structures, Vol. 29, May 10, 2017, pp. 82-90.

9. R. Suzuki, M. Okui, S. Iikawa, Y. Yamada, T. Nakamura, "Evaluation of wearable assistive suit "Airsist I" with variable viscoelastic control strategy", $36^{\text {th }}$ Robotic Society of Japan, Kasugai, Aichi, Japan, September 4-7, 2018, 1A1-01 (in Japanese).

10. S. Iikawa, M. Okui, R. Suzuki, Y. Yamada, T. Nakamura, "Development of variable viscoelastic assistive suit -Effect of the viscosity to human and proposal of variable viscoelastic control strategy-“, Journal of the Robotics Society of Japan, Vol. 36, No. 8, 2018, pp. 49-57 (in Japanese).

11. H. Kawamoto, and Y. Sankai, "Power Assist Method Based on Phase Sequence Driven by Interaction between Human and Robot Suit", in Proceedings of the 2004 IEEE 
International Workshop on Robot and Human Interactive Communication Kurashiki, Okayama, Japan, September 20-22, 2004, pp. 491-496.

12. John Paul Varkey, Dario Pompili, and Theodore A. Walls, "Human motion recognition using a wireless sensor-based wearable system", Personal and Ubiquitous Computing (2012) 16:897-910, DOI 10.1007/s00779-011-0455-4.

13. Andrea Mannini, and Angelo Maria Sabatini, "Machine Learning Methods for Classifying Human Physical Activity from On-Body Accelerometers", Sensors 2010, 10, 1154-1175, DOI 10.3390/s100201154.

14. Atsushi Tsukahara, Yasuhisa Hasegawa, and Yoshiyuki Sankai, "Standing-Up Motion Support for Paraplegic Patient with Robot Suit HAL", in Proceedings of the 2009 IEEE International Conference on Rehabilitation Robotics, Kyoto International Conference Center, Japan, June 23-26, 2009, pp.211-217.

15. Q. Huang, K. Kaneko, K. Yokoi, S. Kajita, T. Kotoku, N. Koyachi, H. Arai, N. Imamura, K. Komoriya, K. Tanie, "Balance Control of a Biped Robot Combining Off-line Pattern with Real-time Modification”, in Proceedings of the 2000 IEEE International Conference on Robotics \& Automation, San Francisco, CA, USA, April 2000, pp. 3346-3352.

16. K. Sano, E. Yagi, and M. Sato, "A Study on Estimation of Walking Intention Using Foot Switches and Hip Joint Angles for Walking Assist of Non-handicapped Persons", in Proceedings of the SICE Annual Conference 2013, Nagoya, Japan, September 14-17, pp. 1527-1532.

17. Misako Sasayama, Toshiyuki Murakami, “A Study on Controller Characteristics for Most Efficient Gait Assist System", IEEE Journal of Industry Applications, Vol. 3, No. 3, pp. 206-213, 2014. 